Revue de l'Institut des langues et cultures

d'Europe, Amérique, Afrique, Asie et Australie

$32 \mid 2018$

Didactique des langues et cultures de spécialité : méthodes, corpus et nouvelles technologies

\title{
Les stratégies linguistiques et les perspectives pour le français sur objectif spécifique en Serbie
}

The Linguistic Strategies and Perspectives for the French for Specific Purpose in Serbia

\section{Danjiel Živković}

\section{OpenEdition}

Journals

Édition électronique

URL : http://journals.openedition.org/ilcea/4788

DOI : $10.4000 /$ ilcea.4788

ISSN : 2101-0609

Éditeur

UGA Éditions/Université Grenoble Alpes

Édition imprimée

ISBN : 978-2-37747-059-4

ISSN : $1639-6073$

Référence électronique

Danjiel Živković, «Les stratégies linguistiques et les perspectives pour le français sur objectif

spécifique en Serbie », ILCEA [En ligne], 32 | 2018, mis en ligne le 01 juillet 2018, consulté le 01 mai 2019. URL : http://journals.openedition.org/ilcea/4788 ; DOI : 10.4000/ilcea.4788

Ce document a été généré automatiquement le 1 mai 2019.

(c) ILCEA 


\section{Les stratégies linguistiques et les perspectives pour le français sur objectif spécifique en Serbie}

The Linguistic Strategies and Perspectives for the French for Specific Purpose in Serbia

Danjiel Živković

\section{Introduction}

Dans les conditions de l'économie mondialisée, la distance ne joue plus un rôle important, par contre la concurrence est devenue plus sévère de sorte que seules les entreprises qui développent une meilleure gestion internationale et qui trouvent les stratégies clés d'entrée sur les marchés étrangers peuvent survivre (Mayrhofer \& Urban, 2011:64). Après l'identification de l'offre d'exportation (produits ou services), l'entreprise devrait identifier le marché étranger et ensuite définir sa stratégie pour le conquérir, trouver le meilleur canal de vente, de la distribution, de la logistique, et connaître davantage l'environnement culturel (Lemaire, $2013: 281$ ). Selon Grin, « [...] tout ce qui relève du marketing, de la vente et du service après-vente exige, à des degrés divers, un travail de communication qui passe par la langue » (2013:7). Par ailleurs, pour avoir du succès sur le marché étranger, la gestion internationale doit dans l'idéal tenir compte des caractéristiques sociales et culturelles d'une société et bien sûr connaître la langue, comme étant l'un des composants de base d'une culture. En prenant connaissance de la langue et de la culture il est donc possible de comprendre les valeurs et attitudes influençant le comportement des personnes de certains pays, ce qui est crucial dans les affaires.

Il est évident que la langue anglaise, au cours des dernières décennies, a émergé comme la lingua franca, et sa connaissance se considère indispensable dans l'économie internationale. Pourtant, la connaissance de la langue anglaise uniquement ne peut pas 
couvrir les spécificités de chaque culture, car les caractéristiques culturelles se transmettent principalement par les langues maternelles ou lingua culturalis. Par conséquent, les entreprises qui envisagent de s'intégrer avec succès sur le marché francophone, devraient dans leurs planifications stratégiques anticiper également l'étude de la langue et de la culture française. Bien qu'à première vue l'apprentissage de la langue française peut sembler extrêmement difficile, il est possible de maîtriser la langue dans un très court laps de temps en appliquant la démarche FOS (Mangiante \& Parpette, $2004: 6)$.

En fin de compte l'entreprise a besoin d'acquérir sans cesse de nouvelles connaissances pour pouvoir maintenir la course avec la concurrence. D'ailleurs, l'apprentissage et la diffusion des connaissances entre les employés sont essentiels à la réussite des entreprises sur le marché, et cela pourrait être certainement appliqué à l'apprentissage d'une langue étrangère. Il se trouve que l'apprentissage organisationnel est également un moyen important d'obtenir un avantage concurrentiel. Étant donné que les hommes d'affaires en Serbie sous-estiment généralement l'importance des connaissances dans la langue française pour se placer sur le marché français, on considère qu'il est d'abord nécessaire de présenter les enjeux des stratégies linguistiques pour la réussite de l'entreprise. Par conséquent, l'objectif de cet article est de mettre en évidence le rôle des stratégies linguistiques dans les affaires et d'examiner les perspectives de la démarche FOS en Serbie.

4 De ce fait, dans un premier temps, nous présenterons le rôle du multilinguisme dans les affaires et les avantages que l'entreprise aurait dans l'application des stratégies linguistiques, ainsi que les études de l'UE concernant le multilinguisme. Dans un second temps, après une brève description de l'état actuel des relations économiques francoserbes, nous examinerons les perspectives de la démarche FOS en Serbie par l'analyse quantitative des données recueillies grâce à une enquête, menée auprès des entreprises, concernant la nécessité de la langue française dans leurs affaires avec le marché francophone.

\section{Importance des stratégies linguistiques}

5 Selon Kotler et ses collaborateurs (2005: 49), la stratégie est un ensemble de décisions et d'activités visant à atteindre les objectifs d'une organisation. En d'autres termes, les entreprises qui utilisent la gestion stratégique ont généralement une meilleure performance dans les affaires, puisqu'elles sont obligées d'examiner tous les aspects de l'entreprise. Par ailleurs, la gestion stratégique a pour objectif d'avoir un avantage sur la concurrence. Pourtant, les produits et les services ne représentent plus l'avantage unique de la concurrence, mais la possibilité d'acquérir de nouvelles connaissances et de cette façon s'adapter aux changements fréquents des marchés.

La stratégie est l'orientation des activités d'une organisation à long terme. Elle consiste à obtenir un avantage concurrentiel grâce à la reconfiguration des ressources de l'organisation dans l'environnement changeant, afin de répondre aux besoins du marché et aux attentes des différents parties prenantes (propriétaires, employés, financeurs, etc.). (Johnson \& Scholes, $2000: 27)$

6 Les stratégies linguistiques, c'est-à-dire les stratégies de gestion des langues font partie de la gestion stratégique d'une organisation. Selon l'étude de l'UE Effects on the European Economy of Shortages of Foreign Language Skills in Enterprise (ELAN), les stratégies 
linguistiques se définissent comme "l'adoption planifiée d'une gamme de techniques visant à permettre une communication efficace avec les clients et les fournisseurs à l'étranger" (Commission européenne, 2006:5). De plus, le forum des entreprises européennes sur le plurilinguisme Les langues font nos affaires donne également des exemples d'entreprises ayant connu le succès grâce aux stratégies linguistiques. Voici quelques-uns des avantages que les entreprises pourraient avoir avec l'application des stratégies linguistiques:

\subsection{La condition de base pour se placer sur le marché étranger}

7 La première étape dans la gestion du commerce extérieur est l'établissement des relations d'affaires avec les clients étrangers, qui peuvent être obtenus grâce aux foires commerciales, aux missions ou aux médias, ce qui est impossible sans la connaissance de la langue. De plus, lorsqu'il s'agit de la conquête des marchés étrangers, l'une des conditions est la connaissance de la langue et la culture de ce marché (Živković, 2016 : 147-148). De cette façon, l'entreprise montre son respect envers l'identité et la culture de ses clients, elle obtient une meilleure compréhension des lois et des coutumes locales, elle donne la preuve d'un engagement à long terme et par conséquent elle augmente la bonne volonté et la confiance, qui sont d'une très grande importance aujourd'hui. Mayrhofer et Urban déclarent: «La culture occupe une place centrale dans le champ du management internationale. Elle concerne toutes les entreprises qui mènent les activités à l'étranger, quels que soient leur taille et leur degré d'internationalisation. » (2011 : 209)

\subsection{Avantage par rapport à la concurrence}

La gestion stratégique permet à l'entreprise de développer et de maintenir un avantage concurrentiel en ce qu'elle a quelque chose que les autres n'ont pas ou bien qu'elle connaît quelque chose de plus que les autres. Donc, l'entreprise ayant un cadre qui maîtrise les langues étrangères a également un atout important par rapport à la concurrence. L'idée principale du Forum des entreprises sur le multilinguisme est que les entreprises ayant la capacité d'avoir des activités professionnelles en plusieurs langues soient plus compétitives et aient plus de possibilités d'accéder à de nouveaux marchés dans un monde globalisé (Commission européenne, 2008). Le succès final d'une entreprise dépend de sa capacité de s'adapter à la société qui créée ses valeurs. Par conséquent, l'entreprise pourrait accroître nettement le succès sur le marché étranger si elle prend connaissance de la langue et de la culture de ses clients.

L'entreprise compétitive est celle qui aura su apprendre, accroître des compétences, innover mieux, plus vite et davantage que ses concurrents actuels et potentiels. Pour assurer la croissance et son développement à long terme, il lui faut favoriser en permanence des comportements d'anticipation (la pensée stratégique) et non se limiter a des comportements d'adaptation, certes indispensables pour réagir aux événements, mais insuffisants pour assurer la pérennité. (Elien, $2015: 2$ )

\subsection{La barrière langagière et culturelle}

Les mots ont une grande puissance dans le travail, de sorte qu'aujourd'hui, il n'existe presque pas de métier où la communication ne joue pas un rôle important. Dès lors, il en résulte que le problème le plus commun dans la gestion internationale est la barrière 
langagière et les différences culturelles. Les responsables des ventes pourraient se trouver dans une position défavorisée s'ils ne parlent pas la langue du client local. Les négociations, les échanges d'opinions, les discussions, les débats, l'harmonisation des intérêts contradictoires, la recherche d'un compromis sont impossibles sans une bonne maîtrise de la langue. Les plus gros problèmes ressortent également des différences culturelles fondamentales telles que l'histoire, les traditions, les valeurs et les croyances religieuses. Pour se confronter à ces défis, l'entreprise devrait disposer d'un personnel ayant des compétences langagières et interculturelles. Selon les études d'ELAN, les entreprises ont recours à diverses formes de stratégies des langues pour aborder les problèmes dans la communication professionnelle internationale. En outre, $69 \%$ des employeurs croient que les employés dans leurs entreprises ont besoin d'acquérir ou d'améliorer leurs connaissances des langues étrangères (Projet CMC_E).

\section{Les études de l'UE concernant le multilinguisme}

10 Afin de développer le multilinguisme, en tant que facteur de croissance économique, l'UE a lancé plusieurs études et projets :

- ELAN (Effects on the European Economy of Shortages of Foreign Language Skills in Enterprise) est la première étude sur la perte des marchés et des occasions d'affaires, en raison du manque de connaissance des langues étrangères. Son objectif était de présenter des informations pratiques et des analyses sur l'utilisation de la langue dans les petites et les moyennes entreprises (PME). L'étude était basée sur un échantillon de 2000 PME des États membres et des pays candidats. Selon les résultats de cette étude, $11 \%$ des PME européennes (soit environ 945000 entreprises) ont perdu un marché en raison de la méconnaissance des langues étrangères et des cultures. Par contre, les entreprisses ayant investi dans la mise en œuvre de stratégies linguistiques ont augmenté leurs exportations de 44,5\% et leurs productivités jusqu'à $3,7 \%$ par rapport à celles qui n'ont pas fait cet investissement (Commission européenne, $2006: 8$ ).

- PIMLICO (Promoting, Implementing, Mapping Language and Intercultural Communication Strategies ) est un projet basé sur les études ELAN avec l'objectif de promouvoir des stratégies linguistiques dans les PME. Ce projet décrit les meilleurs modèles d'application des stratégies linguistiques dans les $40 \mathrm{PME}$ européennes ayant connu une croissance économique (Commission européenne, 2011).

- DYLAN (Language Dynamics and Management of Diversity) est un projet réalisé dans la période de 2006 à 2011 et qui a réuni 19 institutions de 12 pays. L'objectif de ce projet est l'identification des conditions dans lesquelles la diversité linguistique de l'Europe peut être un atout pour le développement des connaissances et de l'économie.

- CELAN (Language Strategies for Competitiveness and Employability) est une étude ayant comme objectif principal d'améliorer la compétitivité et l'employabilité grâce à des stratégies d'apprentissage des langues. L'étude CELAN a fait un sondage parmi 484 entreprises pour étudier leurs besoins langagiers et faciliter la communication entre les acteurs économiques et les linguistes. Selon cette étude $90 \%$ des entreprises ont déclaré qu'elles ont besoin de la connaissance des langues étrangères pour le fonctionnement et pour la compétitivité sur le marché (Cotance, $2011: 11$ ).

- LINCQ (Les langues dans la qualité des entreprises) est un projet financé par le Centre européen pour les langes vivantes (CELV). L'objectif principal du projet est de promouvoir le plurilinguisme au sein des entreprises, ainsi que la réalisation des outils pour la 
reconnaissance des systèmes d'évaluation de la compétence linguistique. Par ailleurs, l'équipe du projet cherche à inclure la compétence linguistique dans des programmes de qualité (Piccardo \& Ortiz, 2013).

- LEMP (Langues et employabilité) est le projet lancé en 2014 qui vise à obtenir un panorama des besoins des employeurs (Peskine, 2015).

11 Pourtant, dans de nombreux pays et entre autres en Serbie également, la politique des stratégies linguistiques n'est pas suffisamment développée ou n'est pas du tout développée. En conséquence, nous jugeons approprié que les entreprises doivent d'abord prendre connaissance de ces faits et ainsi, nous avons organisé des tables rondes sur ce sujet.

\section{Les tables rondes sur les stratégies linguistiques}

12 En tenant compte des faits ci-dessus, on peut facilement arriver à la conclusion que les entreprises envisageant de se rendre sur le marché francophone devrait disposer d'un personnel parlant français et interculturellement compétent. L'accélération de la mondialisation et des échanges économiques entrainent la nécessité de professionnels maîtrisant la langue française. C'est pourquoi le FOS représente aussi l'une des priorités du ministère des Affaires étrangères (Mangiante \& Desroches, 2014 : 52). À ce titre, afin de présenter ces faits aux entreprises serbes nous avons organisé trois tables rondes ${ }^{1}$ intitulées Pourquoi développer une stratégie linguistique? en collaboration avec l'Institut français de Serbie, la Chambre de commerce et d'industrie France Serbie (CCIFS), le club d'affaires belge BSBA (Belgian Serbian Business Association), qui ont eu lieu dans les chambres de commerce des plus grandes villes de Serbie. Le public visé était les cadres, les DRH, les représentants des chambres de commerce chargés des relations économiques internationales, etc. De même, un grand nombre de journalistes et des médias était intéressé par ces événements (voir l'image 1). 
Image 1. - La table ronde Pourquoi développer une stratégie linguistique?

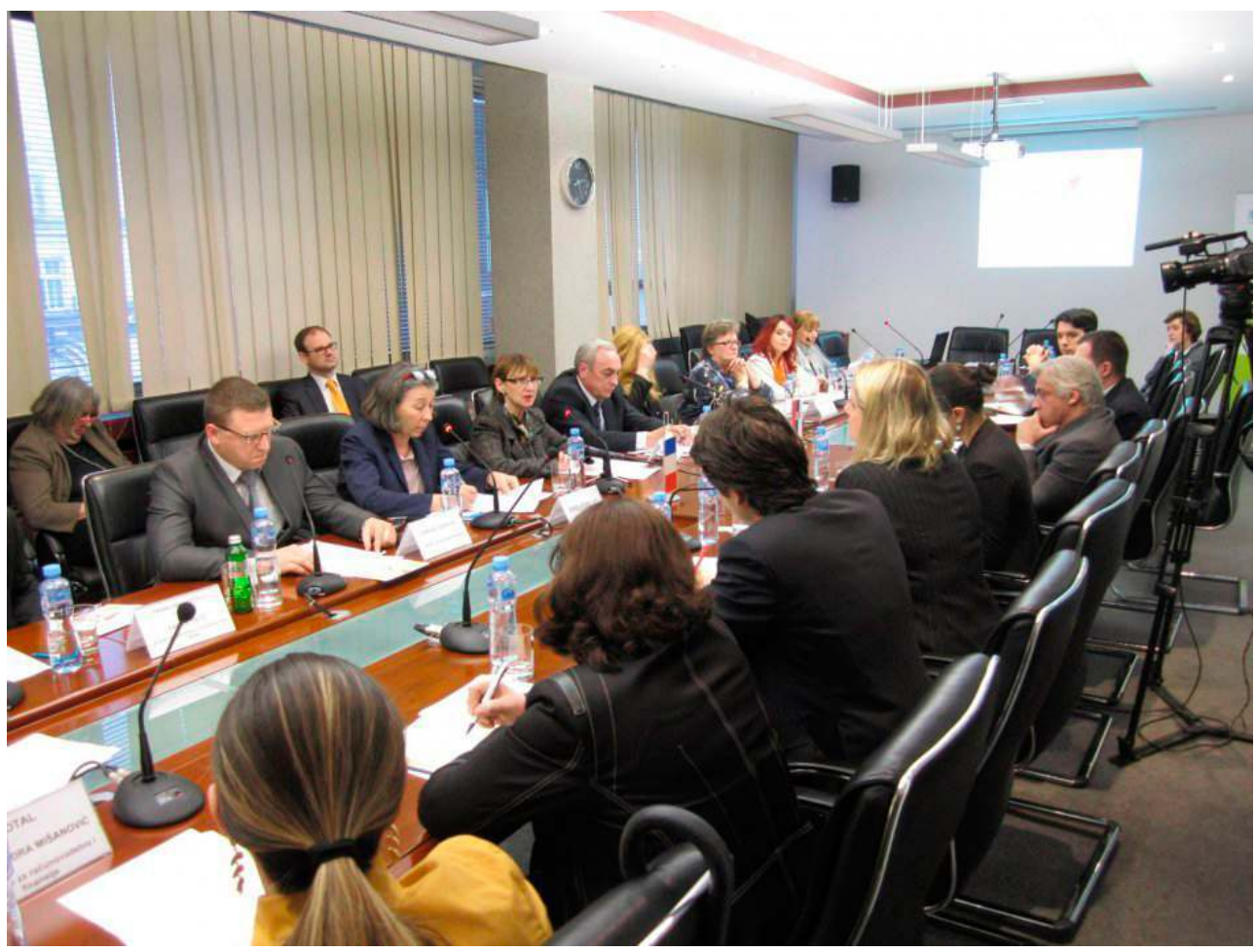

Source : <https://rs.ambafrance.org/francophonie-economique-strategie-langue-francaise-au-servicedes-entreprises>

13 L'objectif principal était de démontrer aux entreprises serbes les avantages qu'elles pourraient avoir en appliquant les stratégies linguistiques et surtout les stratégies d'apprentissage de la langue française pour traiter avec les partenaires francophones. Chaque table ronde était organisée en trois temps :

1. La démonstration des enjeux d'une stratégie linguistique pour les entreprises. Nous avons expliqué aux employés l'importance de l'application des stratégies linguistiques et ainsi l'importance de la connaissance de la langue française pour les activités professionnelles sur le marché francophone.

2. Les témoignages des DRH, cadres, et commerciaux ayant une expérience au sein de leurs entreprises, en lien avec la langue française. La plupart des employés ont admis qu'ils avaient des difficultés dans la communication professionnelle en raison de l'ignorance de la langue et de la culture et qu'ils étaient intéressés d'améliorer leurs compétences linguistiques.

3. La proposition de solutions pour les entreprises en Serbie. Étant donné que l'idée subsiste que la langue française est difficile et que l'entreprise dépenserait beaucoup de temps et d'efforts pour apprendre la langue, nous avons présenté la méthode FOS, exclusivement pour les besoins de l'entreprise dans un délai très court.

\section{Les perspectives pour le FOS en Serbie}

14 Après plusieurs années de stagnation, les relations franco-serbes sont depuis quelques années sur la bonne voie. Il convient d'ajouter que de bonnes relations franco-serbes existent depuis deux siècles, ce qui constitue une base solide pour le développement de la 
coopération économique. La Serbie est située au centre des Balkans occidentaux, ce qui lui permet d'avoir une position stratégique. De plus, les nombreux accords de libreéchange tels que CEFTA, UE, AELE et la fiscalité attractive, présentent la Serbie comme étant un marché attrayant. Ces faits sont certainement intéressants pour les investisseurs français qui considèrent la Serbie comme un partenaire stratégique (CCI Paris Île-deFrance). Dans la période 2000-2011, ils ont investi environ 500 millions d'euros d'investissements directs, et si l'on tient compte de l'investissement individuel des entreprises françaises, les investissements atteignent 850 millions d'euros. Une centaine d'entreprises françaises sont déjà installées en Serbie et elles emploient environ 11000 personnes. De plus, de bonnes expériences des hommes d'affaires français en Serbie représentent aussi un atout. Selon André Marcon, président de la CCI France, «la Serbie, c'est en quelque sorte le hub économique de la région des Balkans occidentaux » (CCIFS). Nous ne mentionnons que quelques données de l'ambassade de France en Serbie illustrant l'état actuel des relations économiques bilatérales entre les deux pays :

- La Serbie est la première destination des investissements français dans la zone des Balkans occidentaux. Le volume des échanges commerciaux a augmenté de manière significative : avec 750 M€ en 2015, il était $20 \%$ plus élevé qu'en 2014 et 80 \% plus élevé qu'en 2010.

- Business France a organisé le 15 septembre 2016 à Paris une journée d'information et de contacts sur la Serbie. Lors de cet événement les atouts de l'économie serbe sont présentés aux entreprises françaises.

- Le forum d'affaires sur les Balkans occidentaux a réuni les ministres de l'Économie de la région, les représentants de l'Union européenne et des institutions financières européennes le 4 juillet 2016 à Paris. Lors de ce forum, les participants ont eu l'opportunité d'échanger des idées autour de trois tables rondes sur les thèmes des infrastructures de transport, de l'énergie/environnement et du numérique.

- En octobre 2016, une nouvelle usine de l'entreprise française Hutchinson, la filiale du géant pétrolier français Total a été ouverte.

- En mars 2016, Michelin a achevé l'extension de l'usine Tigar, sa filiale à Pirot.

Par ailleurs, les entreprises des pays francophones installées en Serbie n'expriment généralement pas le besoin de développer une stratégie RH en faveur de l'apprentissage de la langue française, la langue de travail étant le plus souvent le serbe ou même l'anglais dans leurs relations avec leur siège pourtant francophone. Des initiatives individuelles émergent cependant qui mettent en avant l'enjeu professionnel en termes de carrière, d'évolution au sein de l'entreprise, reconnaissant que la connaissance du français est un atout, signe d'un engagement au sein de l'entreprise, témoignage d'une certaine forme de loyauté. Pour les entreprises serbes, il en est tout autrement. Pour elles, l'intérêt d'une stratégie de formation des employés, notamment commerciaux en langue française est plus qu'évident pour s'ouvrir aux marchés francophones où la langue anglaise ne suffit pas.

Même si en comparant les indicateurs macro-économiques on remarque une grande différence concernant la surface, la population et le niveau de développement des deux pays, le potentiel pour l'amélioration des relations économiques existe. Il convient d'ajouter qu'il existe un grand intérêt des entreprises serbes pour le marché français et pour le marché francophone ${ }^{2}$ en général. Selon les données de la Chambre de commerce serbe (CCS), l'exportation de la Serbie en France a augmenté de 17,5\% par rapport à 2014, et elle s'est élevée à presque 370 millions d'euros. Jusqu'en 2013, la France était le treizième partenaire en termes d'exportations et depuis 2014, elle est devenue le dixième 
pays partenaire dans les exportations totales de la Serbie. Les atouts sont avant tout dans les secteurs de l'agro-alimentaire. Ce marché se développe de façon intensive en France avec un taux de $19 \%$ par an, ce qui représente une grande opportunité pour les producteurs certifiés. En outre, une grande opportunité se trouve dans l'industrie des technologies d'informations et dans le secteur des nouvelles technologies. Pour améliorer ses résultats, la Serbie devrait travailler à la promotion de l'économie auprès des investisseurs français. Pourtant, dans une certaine mesure, l'entrée des produits serbes sur le marché français est empêchée à cause d'une faible connaissance de la langue française dans les milieux professionnels (Živković, Božić Miljković \& Mihajlović, $2017: 48)$.

\section{Les résultats de la recherche}

17 Dès lors, pour les besoins de la recherche, une enquête a été menée auprès des participants des tables rondes afin de préciser la nécessité de la langue française pour les entreprises en Serbie. Le groupe cible était les entreprises ayant des contacts professionnels avec les marchés francophones. L'objectif de l'enquête était de déterminer les problèmes concernant la connaissance de la langue et de la culture des partenaires francophones. L'étude a inclus 42 entreprises dont, 11 entreprises avec moins de 10 employés, 12 entreprises qui ont entre 10 et 50 employés et 19 entreprises qui ont plus de 50 employés. La question de l'enquête est indiquée dans le graphique 1 sur l'axe horizontal (voir l'annexe et le graphique 1). L'enquête était composée de quatorze questions dont huit questions fermées pour lesquelles les participants devaient entourer oui (la couleur fuchsia sur le graphique 1) ou non (la couleur turquoise sur le graphique 1), selon qu'ils étaient d'accord avec la déclaration ou non, quatre questions à choix multiples et deux questions qui exigeaient des courtes réponses. Les réponses de l'enquête en pourcentage sont indiquées dans le graphique 1 sur l'axe vertical. L'hypothèse de l'enquête était que les entreprises en Serbie manquent d'un personnel parlant la langue française et connaissant la culture française ou la culture des autres pays francophones. L'inconvénient de cette enquête est un nombre insuffisant d'entreprises ayant participé. La raison est que certaines entreprises ne pouvaient participer aux tables rondes en raison des engagements qu'elles avaient ces jours-là, ainsi que la non-participation des entreprises provenant de plus petites villes de Serbie. Cependant, on considère que cela ne changerait pas de manière significative les résultats de l'enquête parce que nous avons eu l'occasion d'entrer en contact avec des entreprises souhaitant fortement y assister, mais qui en ont été empêchées, et de discuter du rôle que joue la langue française dans leurs affaires avec le marché francophone. En outre, un grand nombre d'entreprises qui n'assistaient pas à ces tables rondes pour diverses raisons, sont des membres de la Chambre de commerce et d'industrie France Serbie ; ainsi avons-nous eu l'occasion d'entendre leurs opinions sur cette problématique et d'en venir à la conclusion qu'ils sont très proches des entreprises ayant participé à l'enquête.

Les résultats de l'enquête ont montré que presque tous les participants, soit $93 \%$, sont d'accord sur le fait que les employés de leurs entreprises ont besoin d'améliorer la connaissance des langues étrangères. Il est intéressant de remarquer que même si 73 \% des entreprises ont des employés qui parlent français, $93 \%$ ont déclaré que la Serbie manque de personnel professionnel parlant français (voir le graphique 1). La raison de ce résultat pourrait être que la connaissance de la langue française des employés est 
insuffisante ou inadéquate. Ces données dénoncent également le fait que l'enseignement de la langue française en Serbie devrait être plus centré sur les besoins de la société, des institutions et des entreprises. Il est possible d'arriver à la même conclusion après avoir analysé les réponses qui ont été données à la question «Quel est l'obstacle principal pour les entreprises serbes à se placer sur le marché francophone?» Les réponses les plus fréquentes étant les barrières linguistiques, l'ignorance de la langue française et le manque de connaissances culturelles du marché francophone.

Graphique 1. - Les résultats de l'enquête.

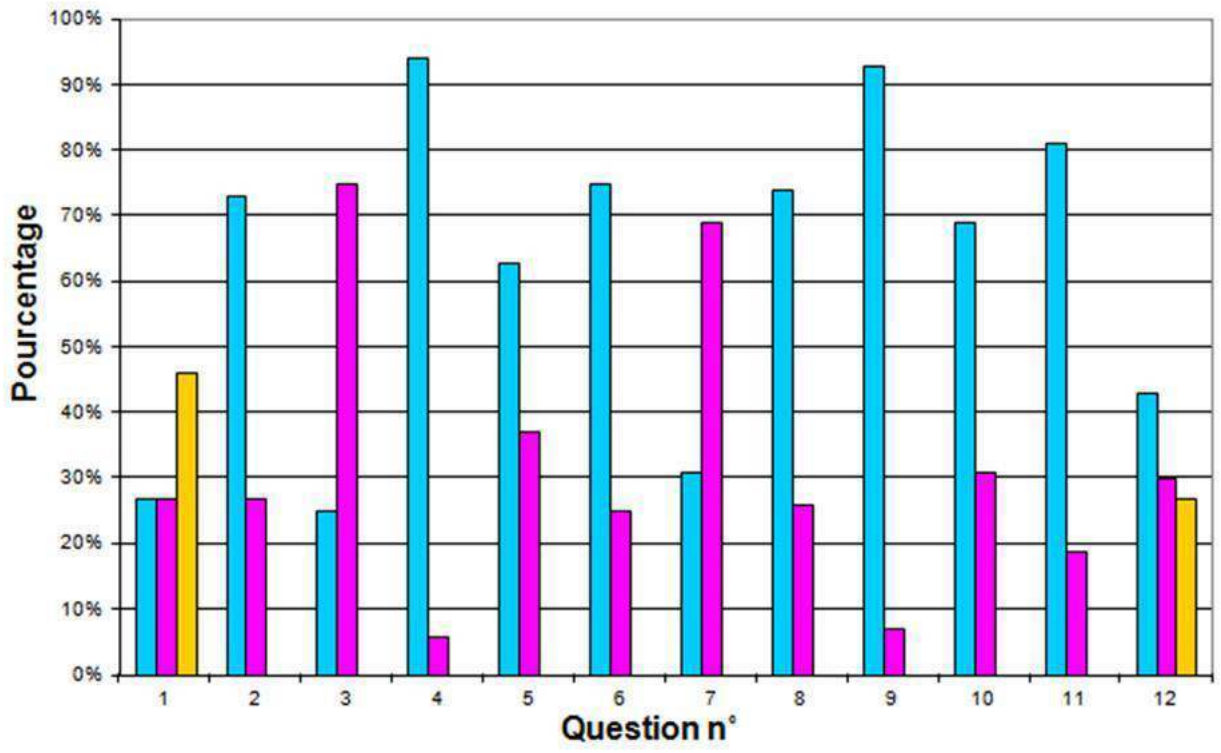

Presque tous les participants considèrent que l'employé parlant plusieurs langues est important pour l'entreprise dont $69 \%$ le considérant très important et $31 \%$ le considérant important. Personne n'a répondu que l'employé qui parle plusieurs langues a peu d'importance. La grande majorité des participants $(62,5 \%)$ ont déjà eu une coopération professionnelle avec des entreprises du monde francophone et $75 \%$ envisagent d'établir une coopération professionnelle avec des entreprises en France à l'avenir. Cependant, seulement $31 \%$ ont visité des foires ou des expositions en France. La raison de ce mauvais résultat est justement la connaissance insuffisante de la langue française par les employés, car comme nous l'avons déjà mentionné dans ce travail, l'établissement de bons contacts professionnels aux foires commerciales est presque impossible sans la connaissance de la langue. Cette hypothèse est étayée également par le fait que, pendant la conversation avec les participants des tables rondes, la plupart ont admis qu'il était très difficile de se présenter à la foire ou au salon sans la connaissance de la langue du pays. Pourtant, la compréhension des relations interculturelles dans l'économie est très importante pour $43 \%$ des participants, pour $30 \%$, elle est importante et même $27 \%$ considèrent que c'est peu important pour les affaires. Une telle réflexion peut être due au fait que les entreprises manquent d'informations sur l'importance de la compétence interculturelle dans l'économie. Par ailleurs, seulement $25 \%$ des entreprises ont envoyé des employés en formation professionnelle en France. À part la langue anglaise, essentiel pour le succès dans les affaires, les participants ont mentionné généralement l'allemand, le français et le russe. À la question « Dans quel secteur croyez-vous que les compétences linguistiques sont nécessaires?», $43 \%$ des participants considèrent que c'est le 
management (la couleur turquoise sur le graphique 1), $30 \%$ la vente (la couleur fuchsia) et $27 \%$ le marketing (la couleur jaune).

Les résultats de l'enquête ont clairement montré qu'en Serbie il existe un besoin d'une connaissance approfondie de la langue française. Nous considérons que l'application de la démarche FOS pourrait contribuer à la résolution de problèmes linguistiques et culturels auxquels sont confrontées les entreprises en Serbie dans les affaires avec le marché de langue française. Cependant, même si la langue française est étudiée dans les quatre universités de Serbie ${ }^{3}$, aucune université ne propose des cours de FOS. Entre autres, il est nécessaire d'influencer les institutions éducatives, principalement les universités à prendre en compte les besoins linguistiques des entreprises en Serbie, ainsi que de former le cadre compétent pour appliquer la méthode FOS. En appliquant les indications fournies par l'UE, à travers divers projets et programmes, nous avons déjà proposé des stratégies à la fois aux niveaux macro et micro (Živković, $2013: 222$ ).

\section{Conclusion}

21 De nombreuses études réalisées au cours des dernières années indiquent clairement que le multilinguisme et l'interculturalisme sont d'une importance capitale pour le management international. La langue est un outil clé dans la communication professionnelle, car il ne peut y avoir d'achèvement des activités commerciales sans communication. Une bonne communication peut augmenter les profits, de la même manière qu'une mauvaise peut les réduire. Les tables rondes ont permis de faire le point sur l'opportunité pour les entreprises d'adopter une stratégie linguistique en lien avec les marchés qu'elles souhaitent conquérir. En établissant des stratégies pour l'enseignement/ apprentissage des langues étrangères, comme étant un des facteurs pour l'amélioration de la gestion internationale, on crée les conditions pour la compétitivité et dans une certaine mesure, la résolution des problèmes de croissance économique. Selon l'étude PIMLICO, les entreprises qui ont mis en œuvre des stratégies linguistiques ont connu des résultats significatifs sur les marchés étrangers et une croissance de la productivité. La confiance est la base de toutes les relations d'affaires et la communication avec le client dans sa propre langue est également un facteur important pour sa construction.

Par conséquent, il est évident que les entreprises qui envisagent de se rendre sur le marché francophone doivent disposer d'un cadre qui maîtrise la langue française. Si l'on tient compte du fait que les relations économiques bilatérales entre la France et la Serbie sont à la hausse et qu'il y a un grand potentiel pour la coopération économique mutuelle, il est évident que le FOS a de grandes perspectives en Serbie. De plus, les résultats de l'enquête ont confirmé le fait que la Serbie manque de cadres ayant la capacité de mener une communication professionnelle en français. En favorisant la démarche FOS qui répond le mieux aux besoins des entreprises, il est possible d'offrir des solutions concrètes aux entreprises qui veulent se positionner sur le marché francophone. Bien sûr, il convient de faire des projets qui analyseront sur un nombre plus significatif dans quelle mesure et dans quels domaines les entreprises exigent la connaissance de la langue française. Puis, en coopération avec les chambres de commerce et des institutions d'enseignement, il faudrait d'abord présenter, puis offrir des cours de FOS.

Lorsqu'il s'agit de l'amélioration et de l'adaptation de l'éducation aux marchés financiers, une grande responsabilité repose également sur les établissements éducatifs. En reconnaissant l'importance de la langue et de la culture, le rôle qu'elle peut jouer dans 
l'augmentation des profits et l'amélioration des affaires en général, l'UE a réalisé plusieurs études et projets afin de sensibiliser les cadres professionnels. Évidemment, pour l'enseignant, à côté de la connaissance de la méthodologie du FOS, celui-ci doit aussi connaître le contexte socio-économique de la Serbie, c'est-à-dire la situation sur le marché, les relations bilatérales politiques et économiques entre la Serbie et la France, ainsi qu'avec les autres pays francophones. Dans la période à venir, nous prévoyons d'analyser les usages linguistiques en fonction de la taille de l'entreprise et en fonction des secteurs, ainsi que par rapport au potentiel de la coopération avec les différents pays francophones. Par ailleurs, nous envisageons d'organiser tout d'abord, des tables rondes, principalement pour les entreprises du secteur agro-alimentaire, puisque selon les économistes, c'est dans ce secteur qu'il existe le plus grand potentiel pour la coopération.

\section{BIBLIOGRAPHIE}

COMMISSION EUROPÉENNE (2006), Effects on the European Economy of Shortages of Foreign Language Skills in Enterprise (ELAN), en ligne sur <http://ec.europa.eu/languages/languages-mean-business/files/ elan-fullreport> (7 décembre 2013).

COMMISSION EUROPÉENNE (2008), Les langues font nos affaires. Des entreprises plus performantes grâce à une connaissance accrue des langues. Recommandation du Forum des entreprises sur le multilinguisme, Luxembourg : Office des publications officielles des Communauté européennes, < www.labeleuropeolingue.it/download/documentazione/2012/Raccomandazioni Forum Business e Multilinguismo_2008_fr.pdf> (7 décembre 2013).

COMMISSION EUROPÉENNE (2011), Report on Language Management Strategies and Best Practice in European SMSs: The PIMLICO Project, Tipik et Semantica Ltd, <http://ec.europa.eu/dgs/ education_culture/repository/languages/policy/strategic-framework/documents/pimlico-fullreport_en.pdf> (20 mai 2016).

CotAnCE (2011), Report on Language Needs in Business. Companies' Linguistic \& Language Related Needs in Europe, Language Strategies for Competitiveness and Employability, <www.celan-platform.eu/assets/ files/D1.3-Business_Needs_Report-Final.pdf> (20 mai 2016).

ELIEN Guy (2015), La stratégie d'entreprise, Paris : Clarans consulting.

GRIN Francois (2013), « Plurilinguisme et multilinguisme au travail : le regard de l'économie des langues », Repères Dorif, 4, 29-43, <www.dorif.it/ezine/ezine_articles.php?art_id=136> (17 mai 2017).

Johnson Gerry \& Scholes Evan (2000), Stratégique, Paris : Publi-Union Éditions.

KotLer Philip, Vong Veronica, SAUnders John \& AMStrong Gary (2005), Principles of Marketing, Londres : Pearson Education.

LEMAIRE Jean-Paul (2013), Stratégies d'internationalisation. Nouveaux enjeux d'ouverture des organisations, des activités et des territoires, Paris : Dunod. 
MANGIANTE Jean-Marc \& DESROCHES Franck (2014), « Le FOS un exemple de recherche-action en didactique du FLE », FDLM, 391, 52-53.

MANGIANTE Jean-Marc \& PARPETTE Chantal (2004), Le français sur objectifs spécifiques : de l'analyse des besoins à l'élaboration d'un cours, Paris : Hachette.

MAYRHOFER Ulrike \& URBAN Sabine (2011), Management international : des pratiques en mutation, Paris : Pearson.

Miloević Miladin \& PANTELIĆ Nada (2014), Relations yougoslavo-algériennes de 1956 à 1979, Belgrade : Arhiv Jugoslavije.

PESKINE Laure (2015), « Analyse des besoins des employeurs français au regard des compétences en langues vivantes étrangères. Rapport d'enquête du projet LEMP (Langues et employabilité) ", en ligne sur Association des professeurs de langues vivantes. Les Langues modernes : <www.aplvlanguesmodernes.org/spip.php?article5913> (17 mai 2017).

PICCARDO Enrica \& ORTIZ Isabelle (2013), « Le projet LINCQ du Centre européen pour les langues vivantes », Repères Dorif, 3, <www.dorif.it/ezine/ezine_articles.php?art_id=105>.

Žıvкоvıс́ Danijel (2016), « Le plurilinguisme et l'interculturalisme : les ressources cachées du commerce international », M. Granescu (dir.), Acta technica napocensis : Diversité culturelle et plurilinguisme, 16(4), Cluj-Napoca : UTCN, 143-151.

Žıvкović Danijel, Božıć Miljković Ivana \& MıнAJLović Svetlana (2017), « Knowledge of French Language and Culture: A Precondition for Improving Economic Relations between Serbia and France », Economica, 44(2), 43-53, <www.ekonomika.org.rs/sr/PDF/ekonomika/2017/ clanci17-3/5.pdf>.

\section{Sitographie}

AMBASSADE D'ALGÉRIE EN SERBIE (2016), Les relations économiques entre la république de Serbie et la République algérienne démocratique et populaire, en ligne sur <www.ambserbie-alger.com/francais/ ekonomija - Copie.htm> (17 septembre 2016).

AMBASSADE DE FRANCE EN SERBIE (2016), Économie et commerce, en ligne sur <https:// rs.ambafrance.org/-Economie-et-commerce-> (15 septembre 2016).

BUSINESS FRANCE (2016), Atelier Serbie, en ligne sur <http://export.businessfrance.fr/programmefrance/001PRG-24420+atelier-serbie.html> (28 octobre 2016).

ChAmbre de COMmerce et d'Industrie France SeRbie (2016), Interview : André Marcon, président de la CCI France, en ligne sur <www.ccfs.rs/index.php?id=16753\&tx_news_pi1\%5BnewsID\% 5D=43782\&tx_news_pi1\%5Bnews\%5D=43782\&cHash=3d9d4e1c9709433d3987a805bd8af318> (10 octobre 2016).

CHAMBRE DE COMMERCE SERBE (2016), La coopération avec la France, en ligne sur <www.pks.rs/ MSaradnja.aspx $\mathrm{id}=56 \& \mathrm{p}=1 \& \mathrm{pp}=0$ \&idjezik=1> ( avril 2017).

LA LETTRE DIPLOMATIQUE (2018), Un partenariat stratégique avec la France tourné vers l'UE, en ligne sur $<$ www.lalettrediplomatique.fr/contribution.php?id=41\&idrub=184> (27 juin 2018).

MinISTÈRE DE L'ÉCONOMIE ET DES FINANCES (2016), Forum d'affaires sur les Balkans occidentaux, en ligne sur <www.tresor.economie.gouv.fr/14011_forum-daffaires-sur-les-balkans-occidentaux> (28 octobre 2016).

PRojet CMC_E (2012), en ligne sur <www.cmcproject.it/portale/unitedkingdom/> (28 mars 2012). 
PRoject DYLAN (2006), Dynamique des langues et gestion de la diversité, en ligne sur <www.dylanproject.org/Dylan_fr/presentation/presentation.php> (26 mars 2012).

\section{ANNEXES}

ENQUÊTE. - L'importance de la langue française dans les affaires avec le marché francophone

Nous vous prions de bien vouloir participer à notre enquête et nous aider à déterminer l'importance de la connaissance de la langue et culture française pour les entreprises en Serbie souhaitant se rendre sur le marché francophone. Pour cette enquête, il ne vous faudra pas plus de cinq minutes. L'enquête est absolument anonyme et vous n'avez pas besoin de mettre votre nom. Nous attendons de vous, que vous soyez complètement honnête et de choisir une des réponses offertes en les entourant ou en formulant une courte réponse.

\begin{tabular}{|c|c|c|c|c|c|}
\hline \multirow{2}{*}{1.} & \multicolumn{5}{|c|}{ Quelle est la taille de votre entreprise? } \\
\hline & a. $<10$ employés & b. $10-50 \mathrm{empl}$ & c. > 50 employés & & \\
\hline 2. & \multicolumn{3}{|c|}{ Avez-vous les employés qui parlent français dans votre entreprise? } & oui & non \\
\hline 3. & \multicolumn{3}{|c|}{$\begin{array}{l}\text { Avez-vous envoyé vos employés à la formation professionnelle en } \\
\text { France? }\end{array}$} & oui & non \\
\hline 4. & \multicolumn{3}{|c|}{$\begin{array}{l}\text { Pensez-vous que la Serbie manque de personnel professionnel parlant } \\
\text { français? }\end{array}$} & oui & non \\
\hline 5. & \multicolumn{3}{|c|}{$\begin{array}{l}\text { Avez-vous déjà eu une coopération professionnelle avec des entreprises } \\
\text { du monde francophone? }\end{array}$} & oui & non \\
\hline 6. & \multicolumn{3}{|c|}{$\begin{array}{l}\text { Envisagez-vous dans le futur plan d'établir une coopération } \\
\text { professionnelle avec des entreprises en France? }\end{array}$} & oui & non \\
\hline 7. & \multicolumn{3}{|c|}{ Avez-vous visité des foires ou des expositions en France? } & oui & non \\
\hline 8. & \multicolumn{3}{|c|}{$\begin{array}{l}\text { Lors du recrutement, faites-vous attention aux langues, à part la langue } \\
\text { anglaise, que le candidat parle? }\end{array}$} & oui & non \\
\hline 9. & \multicolumn{3}{|c|}{$\begin{array}{l}\text { Est-ce que les employés de votre entreprise ont besoin d'améliorer leur } \\
\text { connaissance dans des langues étrangères? }\end{array}$} & oui & non \\
\hline \multirow{2}{*}{10.} & \multicolumn{5}{|c|}{ À votre avis, l'employé qui parle plusieurs langues est : } \\
\hline & a. très important & b. important & c. peu important & & \\
\hline \multirow{2}{*}{11.} & \multicolumn{5}{|c|}{ La compréhension des relations interculturelles dans l'économie est... ? } \\
\hline & a. très important & b. important & c. peu important & & \\
\hline
\end{tabular}




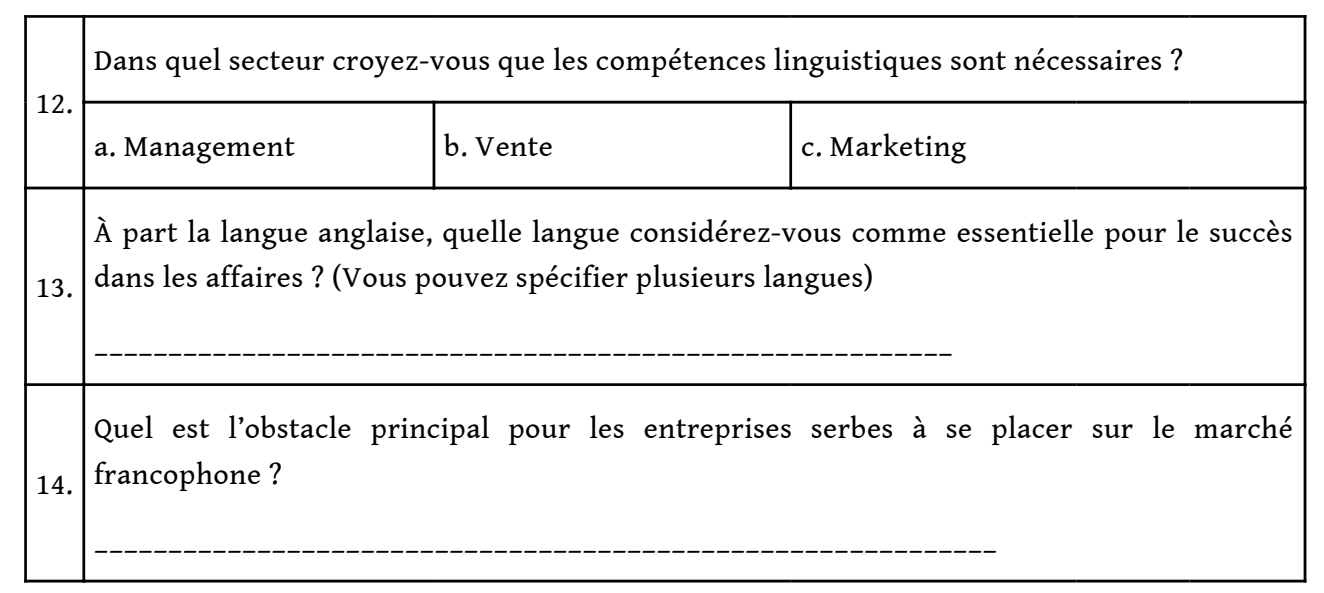

\section{NOTES}

1. À l'occasion du mois de la francophonie, le 21, 23 et 24 mars 2016, trois tables rondes sont organisées dans les chambres de commerce de Belgrade, de Niš et de Novi Sad, autour des enjeux pour les entreprises d'adopter une stratégie linguistique.

2. Parmi les pays francophones, l'Algérie est le pays avec lequel il existe le plus grand potentiel pour la coopération économique si l'on tient compte des relations bilatérales traditionnellement bonnes et de la complémentarité entre les économies des deux pays (Milošević \& Pantelić, 2014). Selon les données de l'ambassade d'Algérie en Serbie, les échanges économiques entre les deux pays s'intensifient à l'époque de Yougoslavie et ils ont atteint une somme de 400 millions $\$$ dans les années 1970. À partir de l'an 2000 les relations bilatérales se renouvellent successivement et en 2005 s'élevaient à 16,3 millions \$ (Ambassade d'Algérie en Serbie, 2016).

3. Le cours de français de spécialité s'enseigne à Niš comme un cours à option.

\section{RÉSUMÉS}

La mondialisation a changé l'environnement des affaires, et il est possible de dire à présent que les marchés potentiels se trouvent dans le monde entier. Pourtant, le problème le plus commun dans la gestion du commerce extérieur et des ventes internationales est l'ignorance des langues étrangères.

Par ailleurs, l'idée que pour le commerce international la connaissance de l'anglais est suffisante semble subsister. Les entreprises méconnaissent bien souvent l'impact qu'une maîtrise de la langue française peut avoir pour leur développement sur les marchés francophones. Dès lors, cet article d'un côté permettra de mettre en évidence le rôle des stratégies linguistiques pour les activités professionnelles et de l'autre côté, en tenant compte de l'état actuel des relations économiques franco-serbes, elle démontrera les perspectives d'apprentissage/enseignement du français sur objectif spécifique (FOS) dans un contexte économique en Serbie.

Nous souhaitons également mener une enquête auprès des entreprises en Serbie afin de déterminer l'importance de la connaissance de la langue et de la culture française pour les entreprises souhaitant se rendre sur le marché francophone. En favorisant la démarche du FOS qui répond le mieux aux besoins des entreprises, nous permettons aux apprenants d'interagir 
plus efficacement dans le monde professionnel. De cette façon nous pourrions également offrir des solutions concrètes aux entreprises qui veulent appliquer la stratégie linguistique et améliorer leurs positions sur les marchés francophones.

Globalization has changed the business environment, and it is now possible to say that the potential markets are all over the world. Yet the most common problem in the management of foreign trade and international sales is the ignorance of foreign languages.

On the other hand, there still seems to be an opinion that for international trade the knowledge of English is sufficient. Companies often misunderstand the impact French language proficiency can have on their development in Francophone markets. This article, on the one hand, will make it possible to highlight the role of language strategies for professional activities and, on the other hand, taking into account the current state of Franco-Serbian economic relations, learning/ teaching of French for specific purpose (FOS) in an economic context in Serbia.

We also want to conduct a survey of companies in Serbia to determine the importance of French language and culture knowledge for companies wishing to appear on the French-speaking market. By promoting the FOS approach that best meets the needs of businesses, we enable learners to interact more effectively in the professional world. In this way we could also offer concrete solutions to companies who want to apply the linguistic strategy and improve their position in the French-speaking market.

INDEX

Mots-clés : stratégies linguistiques, FOS, Serbie, gestion internationale, marché francophone Keywords : linguistic strategies, FSP, Serbia, international management, French-speaking market

\section{AUTEUR}

\section{DANJIEL ŽIVKOVIĆ}

Université Union, Faculté des études de droit et des affaires 